\title{
AUTONOMOUS RECONFIGURATION BY INNOVATION OF DIFFUSIONS
}

\author{
A Distributed Decision-Making Framework for Multi-State \\ Mobile Ad Hoc Networks
}

Timothy K. Forde, Linda E. Doyle and Donal O'Mahony

Centre for Telecommunications Chain-Value Research (CTVR)

University of Dublin, Trinity College, Dublin 2, Ireland.

\begin{abstract}
The spatially and temporally diverse nature of ad hoc networks strongly suggests the development of a sophisticated multi protocol network-layer that should enable the ad hoc network's nodes to dynamically change the routing protocol as networking scenarios demand. In short, the routing protocol is to be considered a system variable. However, the autonomous reconfiguration of such a dependent variable is a non trivial task in the challenging environment of a realistically modelled open, mobile, wireless ad hoc network. We present a novel framework that builds on the social science theory of diffusion of innovations, a theory which describes a flexible decision-making model. This concept enables the development of an autonomous reconfiguration protocol which addresses the constraints imposed by the mobile ad hoc networking system model.
\end{abstract}

Keywords: Distributed Reconfiguration, Autonomic Computing

\section{Introduction}

Ad hoc routing protocols are at the core of ad hoc networks allowing remote nodes to communicate on a peer to peer basis [1], [2], [3]. It is well established in the literature that a one size fits all approach with regard to the choice of optimum routing protocol does not suffice [4]. Rather, in keeping with the distributed and ad hoc nature of these networks, it is preferable to design a system that allows nodes to dynamically configure and utilise the most suitable ad hoc routing protocol [5], [6]. Divergent network scenarios, as exemplified by node mobility, node density and traffic loading, demand networking protocols which are tailored to their requirements. To this end, we propose a flexible and reconfigurable network-layer which has access to a suite of fully distributed ad hoc routing protocols, each protocol addressing a different subset of the set of possible networking scenarios. The routing protocol is a dependent com- 
ponent of the network-layer, i.e. for neighbouring nodes to facilitate communication on a multi-hop peer to peer basis they must operate the same routing protocol. We present a reconfiguration framework that enables the nodes to autonomously and collaboratively choose their network-layer routing protocol having regard to the prevailing network conditions. The decision framework is based on the social science theory of the diffusion of innovations [7]; its design being tightly constrained by the limited applicability of distributed consensus algorithms in an asynchronous message passing system [8].

\section{Network-Layer Reconfiguration}

Framework Objectives. A reconfiguration framework must allow the network, as constituted by autonomous nodes, to react to changes in the network conditions by suitably reconfiguring the network-layer. However, a network is a distributed system; distributed with regard to both its computing architecture and with regard to its physical scope. The geographical spread of an ad hoc network lends itself to the presence of conflicting networking conditions in different areas of the network. Furthermore, robust ad hoc networks employ distributed routing protocols [1], [2], [3] which have been optimised to minimise overhead and to maximise throughput. This inherent networklayer optimisation should not be diminished by the introduction of ancillary network-layer protocols. Also, the reconfiguration protocol should not be reliant on the explicit services of the core network-layer routing protocols as this would introduce a cyclic dependency.

Realistic System Model. In designing a robust network-layer protocol, the traits of a realistically modelled open, mobile, ad hoc network should be borne in mind. Among these traits number the routing protocol signalling profile, the node-to-node link quality and the autonomy of the nodes' mobility. In sum, the ad hoc network can be viewed as a distributed system of nodes which communicate over unreliable message passing channels, the structure of which is subject to dynamic, autonomous change.

\section{Decision-Making: Difficulties in Ad Hoc Networks}

The reconfiguration of the network-layer's routing protocol is a multilateral choice; no single node has innate authority to make a unilateral decision in a fully distributed system in which there are likely to be divergent systems views. The literature on distributed consensus protocols, which is the discipline concerned with the reaching of agreement among remote processes [9], identifies the limits within distributed agreement is possible. The applicability of such protocols is system dependent, varying over a combination of fault, communication, transmission, message ordering and processor assumptions [8]. Any 
other agreement problem may be shown to be at least as hard as distributed consensus [10]. Fischer, Lynch and Patterson proved the impossibility of distributed consensus under certain conditions [11], showing that deterministic distributed consensus is impossible in an asynchronous message passing system if even one process crashes; a crashing process is the simplest form of system failure. To circumvent this result certain remedies, such as the use of failure detectors [12], have been proposed.

Despite the difficulties of reaching consensus in an asynchronous messagepassing system, such as a mobile ad hoc network, distributed consensus has found its way in to the ad hoc networking community through group membership (GM) schemes which enable spontaneous communities to cooperate at application level. Briesemeister et al. [13] suggest a scheme which is based on the notion of local GM. The authors argue that a service designed for mobile ad hoc networks should be partitionable as disconnections may occur frequently. Their localised GM scheme allows an unbounded number of processes to exist, i.e. it allows for an open network. The localised GM service is built around a neighbourhood service that employs a failure detector-like heartbeat mechanism. The heartbeat operates in an asymmetric manner so that neighbouring processes do not have to have to share a symmetric link. The application described in [13] illustrates the narrow scope within which consensus has been applied to mobile ad hoc networking systems, thus far.

A solution to the issue of terminating the decision-making process within the context of a mobile wireless ad hoc network has been presented in [14] which describes an address auto-configuration scheme which adapted the Ricart Agrawala mutual exclusion algorithm for a multi-hop ad hoc network. The scheme uses a soft-state approach, allowing a node to take an address while the mutex-based allocation process is ongoing. If the attempted allocation is denied to the node, or if the node does not receive a confirmation message within a fixed period, then the node relinquishes the address. Termination of the process is thus guaranteed in the presence of lost or delayed confirmation messages.

\section{Diffusion of Innovations: Societal Reconfiguration}

Since the mobile ad hoc network consists of autonomous nodes with varying networking experiences (i.e. link properties, degree of connectedness, and observation of prevailing networking conditions), it is useful to categorise these different types of ad hoc nodes with respect to the part that they would play in the reconfiguration decision making process. To this end, we present a discussion of pertinent elements of the social science concept of diffusion of innovations [7]; a tractable model on which to base a robust reconfiguration 
framework. A simple example of this concept is the adoption of the QWERTY keyboard standard over the ergonomically superior DVORAK keyboard.

Innovation Decision Process. The innovation decision process is the process through which an individual or Decision Making Unit (DMU) passes from first knowledge, or awareness, of an innovation to forming an attitude towards the innovation, to deciding to adopt or reject it, to implementation of the new idea, and to confirmation of this decision. As the process happens over time, the five steps in the process are individually conceptualised as; knowledge, persuasion, decision, implementation and confirmation. Knowledge is the first stage in the innovation-decision process. It occurs when a DMU is exposed to the innovation's existence. Persuasion occurs when the DMU forms a favourable or unfavourable attitude towards the innovation. Individuals seek reinforcement from personal connections and consider the innovation in light of their current situation. As the innovation carries a degree of risk, individuals want to know that their thinking is in line with that of their peers so that it reduces uncertainty about the innovation. During this stage the adoption or rejection of an innovation is not a foregone conclusion. Decision occurs when the DMU engages in activities that lead to either the adoption or rejection of the innovation. DMUs or individuals will often trial an innovation on a probationary period at this stage as part of the decision to adopt. However, in many cases it is not possible to trial the innovation alongside the status quo and so the innovation must either be accepted or rejected in its totality. Implementation occurs when a DMU puts the innovation into use. This stage normally follows directly after the decision stage within a short period of time. The innovation finally loses its distinctive quality as the separate identity of the new idea disappears. Confirmation then occurs when a DMU seeks reinforcement of an innovation that has already been made, but the unit may reverse the decision if it is exposed to conflicting messages about the innovation. In essence, the innovation-decision process is a continual and cyclical process in which the system will look for another innovation if it fails to receive reinforcement about the most recently adopted innovation.

Innovation Decision Actors. The theory goes on to provide useful characterisations of the actors in the innovation-decision process using ideal types. The most popular classificatory principle is based on the innovativeness of the DMU. The literature describes innovativeness as the degree to which an individual or DMU, e.g. a node, is relatively earlier in adopting new ideas than other members of the system. Five adopter categories are defined; innovators, early adopters, early majority, late majority and laggards. Innovators are generally active information seekers about new ideas. They have a high degree of exposure to pertinent information and are eager to try new ideas. The innovator 
has the important role of acting as the gatekeeper that controls the introduction of innovations to other individuals. Early adopters (EA) have the largest degree of persuasive opinion leadership in most social systems; in terms of communication sources, they are localites, i.e their outlook is parochial, based on local observations. Early adopters have a high degree of connectedness and make reasoned, evidence -based decisions. The early adopter has to be more judicious in its innovation-decisions in order to continue to command the respect of others. They decrease local uncertainty about an innovation by adopting it and conveying messages to other near peers by means of interpersonal networks, i.e. neighbour-to-neighbour connections. The early majority (EM) forms a bridge between the early adopters and the late majority. They may deliberate over the adoption of the innovation for a relatively longer period than either the innovator or the early adopter. The early majority do not lead opinion as the early adopter does, but through frequent interaction with their peers, they tend to follow the early adopters. They are crucial in terms of tipping the balance within a society towards adoption of the innovation. They provide interconnectedness between groups of early adopters. The late majority adopt an innovation after the average member of the social system has already done so. The late majority (LM) are often forced to adopt the innovation as a result of economic necessity and increasing social pressures. The late majority, being sceptical, wait until most of their social system have already adopted, i.e. the late majority are more influenced by peer pressure than the utility of the innovation. Laggards (LD), as the name suggests, are the last in a social system to adopt an innovation. They have no opinion leadership qualities and are very local in outlook; in fact many are near isolates in social networks. The point of reference for laggards is the past; their decisions are made in terms of what has been done in previous generations.

Individual Thresholds. An interactive innovation is one for which the utility of the innovation rises as the number of near peer adopters also rises. Individuals are likely to watch the other members of their group in an effort to discern what the group choice may be. The outcome for the group then turns on each member watching what the other members do. A threshold is the number of other individuals who must have adopted an innovation before a given individual will follow. For the diffusion of an innovation, a threshold is reached when an individual is convinced to adopt as a result of knowing that some minimum number of other individuals in the system have also adopted. So, the reaching of systemic critical mass turns on the local actions of individuals with respect to their own thresholds. 


\section{A Self-Stabilizing Network-Layer}

The reconfiguration protocol is supported by a self-stabilizing multi-protocol network-layer built on our communication stack DAWN, the Dublin Ad Hoc Wireless Network [15]. DAWN is a real ad hoc network testbed that facilitates experimentation with ad hoc networks on all levels from the application-layer to the physical-layer. At the network-layer of our communication stack, protocols can be dynamically started up or closed down at runtime, i.e. the state of the node, vis-à-vis its routing protocol, can be configured on the fly. The multi protocol network-layer currently allows a node to use AODV [1], DSR [2] or OLSR [3]. This network-layer also incorporates an ancillary self-stabilising configuration initialisation and conflict resolution protocol [16]. It also incorporates network observation tools that use local information similar to allow nodes to share external-state vectors (ESVs) describing their own neighbourhood observations.

\section{The Reconfiguration Framework}

The protocol operates in three stages; the Persuasion Stage, the Decision Stage and the Implementation Stage. The Reconfiguration Decision Protocol (RDP) extends and modifies elements of the innovation-decision process for the task at hand. The RDP uses a two phase decision-making approach in which nodes are continually in the process of making a soft-state decision and are occasionally asked to make a hard state decision. The component is completely distributed, relying on opportunistic communication between nodes. Even though the system characteristics exacerbate the conditions which underlie the premise for the FLP impossibility result [11], a degree of local decision-making is possible, as exemplified by the local GM scheme [13] and by [14]. The use of such soft-state decisions allows for a more flexible decision-making model. The proposed RDP modifies the general layout of the innovation-decision process format. The distributed consensus model and the innovation-decision model are both triggered when a proposal is made to the system by a single node. In the consensus model the next step generally involves consultation with every other node in the system concluding in a clear termination of the process. In the innovation-decision model, a system of individuals progress from first knowledge though to the persuasion, decision and implementation stages before seeking confirmation of their decision; the confirmation stage may lead to a replacement discontinuance if the DMU is dissatisfied with the innovation. In contrast, the RDP starts in the consultation stage without any proposal being made; we term this the RDP's Persuasion Stage. The knowledge stage is not necessary in this system as the routing protocols are not true innovations; each node is already aware of the protocols. Instead, the system may be seen as one in which three protocols have been 
innovated and the nodes are continually seeking to confirm that their current choice is optimal for the given network conditions.

The Persuasion Stage involves a continuous process of passive observation and analysis. Each node makes soft-state decisions which are based only on passively received information and which may change as that information grows stale and expires. If the nodes manage to sustain a soft-state decision for a time, without altering it, they enter the Decision Stage followed immediately by the Implementation Stage. The Implementation stage completes an execution of the reconfiguration decision protocol and the nodes then re-enter the Persuasion Stage to begin evaluating the networking conditions again.

There are two other general points which should be noted at this stage. Firstly, due to the impossibility of knowing what constitutes the entire network in an ad hoc setting, the reconfiguration happens within a region of the network, i.e. a group of nodes that are sufficiently connected to organise themselves using the network-layer's self-stabilizing configuration conflict resolution protocol [16]. Secondly, the RDP employs small reconfiguration beacons which are appended to all packets that are issued by a node in the course of operating the primary network-layer protocols, i.e. they are opportunistically broadcast. The RDP simply queues its current beacon pending signalling activity by a primary protocol - it never actively issues packets itself.

The Persuasion Stage. It is during the execution of this stage of the protocol that each node decides which protocol suits it, based on the node's knowledge of its local network state. Each node makes decisions based only on information passively received from neighbouring nodes; there is no handshaking. The persuasion stage of the innovation-decision process is concerned with nodes forming an opinion about an innovation; they are either in favour of the innovation or against it. Analogously, nodes in a region of an ad hoc network are continuously engaged in forming an opinion about their preferred ad hoc routing protocol. A node's preferred choice at any given time amounts to a soft-state decision; the node's hard-state decision is reflected in its current working routing protocol. A node's soft-state decision may be in agreement with its current hard-state decision, i.e. its current network-layer configuration. The differentiation of DMUs in the innovation-diffusion process extends to the ad hoc networking context with regard to the nodes involved in the RDP. The notions of Early Adopter, Early Majority, Late Majority and Laggard Nodes are introduced into the RDP to define the decision models used by nodes in the Persuasion Stage. There are no Innovator Nodes as the concept of a knowledge stage does not transfer to this setting. Nodes make their soft-state decisions based on a series of rules that relate to their classifications which are closely analogous to their innovation-decision counterparts. Each node changes the decision-making rules that it uses based on the network observations available 
to it and its knowledge of the decisions of its neighbours. While it is preferable to make a decision based on evidence alone (i.e. EA), nodes are also enabled to make decisions that are influenced by the choices of their neighbours (i.e. EM and LM). For the purpose of clarity note that a node referred to as an Early Majority (EM) Node, for example, is an ad hoc node that has made its decision based on EM decision rules. Both the EM node and the LM nodes' decisions are subject to the control of individual thresholds which dictate how easily persuaded a node can be. For instance an EM node may be required to have a majority of its neighbours as EA nodes before it adopts their decision whereas an LM node may have lower threshold that allows it to adopt the most popular decision that its neighbours have declared. The value to which the thresholds are set dictates the ease with which nodes can agree to reconfigure themselves. Setting the threshold too high would require a high degree of homogeneity within the network whereas lessening the threshold would allow change to occur in more heterogeneous environments. Finally, some other nodes in the system are bound to find themselves with poor connections to the main network partition. These nodes collate observation data that is inconsistent with their neighbours' data and cannot form a decision using one of the above mentioned models. Instead, these LD nodes make no decision. They will follow whatever decision is made by other nodes, if a decision is made.

The reconfiguration beacon has two fields; the Decision field, i.e. which protocol the node prefers, and the Decision Type field which indicates which decision model the node used to arrive at the soft-state decision. The Decision Type field may have the following values; EA, EM, LM, LD and HS (Hard State). The Laggard value indicates that the node could not make a decision and the Hard State value is used to enforce a decision at the Decision Stage.

Each node records information received from neighbours regarding their ESV data and soft-state decision choices in its neighbourhood table. The neighbourhood table is a constituent element of any network-layer's routing protocol; it lists those nodes which to which at least an incoming asymmetric link exists. Entries are added to the table, refreshed and removed in accordance with the techniques associated with the routing protocol in use. Nodes periodically evaluate their recorded observations in order to arrive at a soft-state decision using rules based on the decision hierarchy. This hierarchy places the Early Adopter decision, which is based on network observations alone, at the top and the Laggard decision at the bottom. Each decision is based on the information that is available to the node when it executes an evaluation round. Initial Early Adopter decisions, i.e. the first decisions that can be made in system, are made on the basis of a node's observation that its network observations match those of its neighbouring nodes, which leads them to have a common preferred choice of ad hoc routing protocol. The aim of the reconfiguration protocol is to allow nodes to loosely exchange network observations and 
soft-state decisions that reflect the underlying heterogeneity or homogeneity of their experiences. If the networking conditions are such that neighbouring nodes do not have a common experience of the network, then those nodes will not progress the RDP towards a hard-state decision.

Flushing Stale Data. The accuracy of a node's neighbourhood table varies from routing protocol to routing protocol. A neighbour will only persist in an OLSR cache for a short time before it is expunged or refreshed, whereas a DSR cache is much slower to expunge data. However, the RDP is not concerned with the validity of routing information; it will not be attempting to make use of the out going links. The RDP is only concerned with the associated neighbour entry information such as the ESV data and soft-state-decision data, to provide it with a view of the local network's conditions as seen by neighbouring nodes. If the information persists in the neighbourhood table due to the routing protocol's timeout mechanisms, the RDP may use very stale observation data if it uses every entry in the neighbourhood table with equal weighting. To the RDP, neighbouring nodes are simply a timed series of data sources, nothing else, and it can employ a suitable filter, e.g. an EWMA filter, to place greater weight on the most recently received data and to diminish the effects of data that has not been refreshed.

The Decision and Implementation Stages. The RDP progresses to the Decision Stage when an EA node has been advocating the same decision for a defined period of time. By choosing appropriate time to sustain the EA decision, the RDP can be allowed to progress rapidly through the persuasion stage or it can be made to move slowly towards the Decision Stage. The decisive Early Adopter Node issues a reconfiguration beacon with the Hard State in the Decision Type field. Each receiving neighouring node that has made the same soft-state decision, regardless of how it made the decision, adopts the hardstate decision by changing its protocol. Those nodes then continue to beacon that hard-state decision for a set time. Nodes that are not sufficiently connected are corrected by the configuration conflict resolution protocol [16].

\section{Conclusions}

Given the diverse nature of an ad hoc network it is not a trivial task to enable distributed reconfiguration in a realistically modelled system. We have proposed a novel reconfiguration framework that enables nodes of varying network experiences to collaboratively reconfigure themselves notwithstanding the harsh, yet necessary, conditions that we have imposed. The framework is robust and very low cost, extending the concept of diffusion of innovation to the mobile ad hoc networking domain within the bounds defined by the literature on distributed consensus. While this framework has been developed for 
use at the network-layer, the design conditions that were imposed on it make it suitable for use at the MAC layer or physical layer.

\section{References}

[1] C. E. Perkins and E. M. Royer, Ad-hoc On-Demand Distance Vector Routing, Second IEEE Workshop on Mobile Computing Systems and Applications, 1999, 25-26 Feb. 1999, Pages: $90-100$.

[2] D.B. Johnson and D.A. Maltz, Dynamic Source Routing in Ad Hoc Wireless Networks, In Mobile Computing, edited by Tomasz Imielinski and Hank Korth, Kluwer Academic Publishers, 1996, Pages: 153-181.

[3] T. Clausen, P. Jacquet, A. Laouiti, P. Minet, P. Muhlethaler, A. Qayyum, L. Viennot, Optimized Link State Routing Protocol, IETF MANET Working Group, December 2002.

[4] D.D. Perkins, H.D. Hughes and C.B. Owen, Factors Affecting the Performance of Ad Hoc Networks", Proceeding of the IEEE International Conference on Communications 2002, Vol. 4, Pages: 2048-2052.

[5] J. Boleng, W. Navidi and T. Camp, Metrics to Enable Adaptive Protocols for Mobile Ad Hoc Networks, Proceedings of the International Conference on Wireless Networks, June 24 - 27, 2002, Pages: 293-298.

[6] M. Frodigh, S. Parkvall and C. Roobol, Future-Generation Wireless Networks, IEEE Personal Communications, Vol: 8 Issue: 5, Oct. 2001, Pages: 10 -17.

[7] E.M. Rogers, Diffusion of Innovations, 4rd Edition, Free Press, London.1996.

[8] J. Turek and D. Shasha, The Many Faces of Consensus in Distributed Systems, IEEE Computer, Volume: 25, Issue: 6, June 1992, Pages: 8 - 17.

[9] H. Attiya and J. Welch, Distributed Computing: Fundamentals, Simulations and Advanced Topics, McGraw-Hill Publishing, England, 1998.

[10] R. Guerraoui and A. Schiper, Consensus: The Big Misunderstanding, 6th IEEE Workshop on Future Trends of Distributed Computing Systems, Tunis, Tunisia, October 29 - 31, 1997.

[11] M.J. Fischer, N.A. Lynch and M.S. Paterson, Impossibility of Distributed Consensus with One Faulty Process, Journal of the ACM, Vol. 32, No. 2, April 1985, Pages: 374-382.

[12] T.D. Chandra and S. Toueg, Unreliable Failure Detectors for Reliable Distributed Systems, Journal of the ACM, Volume: 43, Issue: 2, March 1996, Page: 225

[13] L. Briesemeister and G. Hommel, Localized Group Membership Service for Ad Hoc Network, International Conference on Parallel Processing Workshops, August 18 - 21, 2002, Vancouver, B.C., Canada, Page: 94.

[14] S. Nesargi and R. Prakash, MANETconf: Configuration of Hosts in a Mobile Ad Hoc Network, Proceedings of the Twenty-First Annual Joint Conference of the IEEE Computer and Communications Societies., Volume: 2, 23-27 June 2002, Pages: 1059 -1068.

[15] D. O'Mahony and L.E. Doyle, An Adaptable Node Architecture for Future Wireless Networks, in Mobile Computing: Implementing Pervasive Information and Communication Technologies, Kluwer series in Interfaces in OR/CS, Kluwer Academic Publishers, 2002, Pages: 77-92.

[16] T.K. Forde, L.E. Doyle and D. O'Mahony, Self-stabilizing Network-Layer AutoConfiguration for Mobile Ad Hoc Network Nodes, in Proceedings of the IEEE International Conference on Wireless and Mobile Computing, Networking and Communications (Wimob 2005), 22 - 24 August 2005, Montreal, Canada. 